\title{
MODIFICATION OF ANTERIOR APPROACH TO SUPERIOR SULCUS TUMORS
}

\author{
Takamasa Onuki, Masahide Murasugi, Masahiro Mae, Yasuyuki Sone, Junichi Kei, and Sumio Nitta, Tokyo, Japan
}

From The First Department of Surgery, Tokyo Women's Medical University, Tokyo, Japan.

Received for publication Feb 4, 1998; accepted for publication April 9 , 1998.

Address for reprints: Takamasa Onuki, MD, First Department of Surgery, Tokyo Womens' Medical College, Shinjuku-ku, Kawada-chou, Tokyo, 162, Japan.

J Thorac Cardiovasc Surg 1998;116:663-4

Copyright $@ 1998$ by Mosby, Inc.

$0022-5223 / 98 \$ 5.00+0 \quad \mathbf{1 2 / 5 4 / 9 0 8 8 7}$
A combination of radiotherapy and radical resection has become the standard treatment for bronchogenic carcinoma of the superior pulmonary sulcus. When an operative procedure for the anterior type of superior sulcus tumor is applied, especially with large vessel involvement, 2 approaches have been reported. In the first approach, the clavicle is preserved or reconstructed, but with deep narrow exposure. In the second, the clavicle is resected, widening the operative field. In our study, with a modification of the second approach, bone structure, including parts of clavicle, the anterior portion of the first rib, and the parts of the sternum were preserved and reconstructed. 

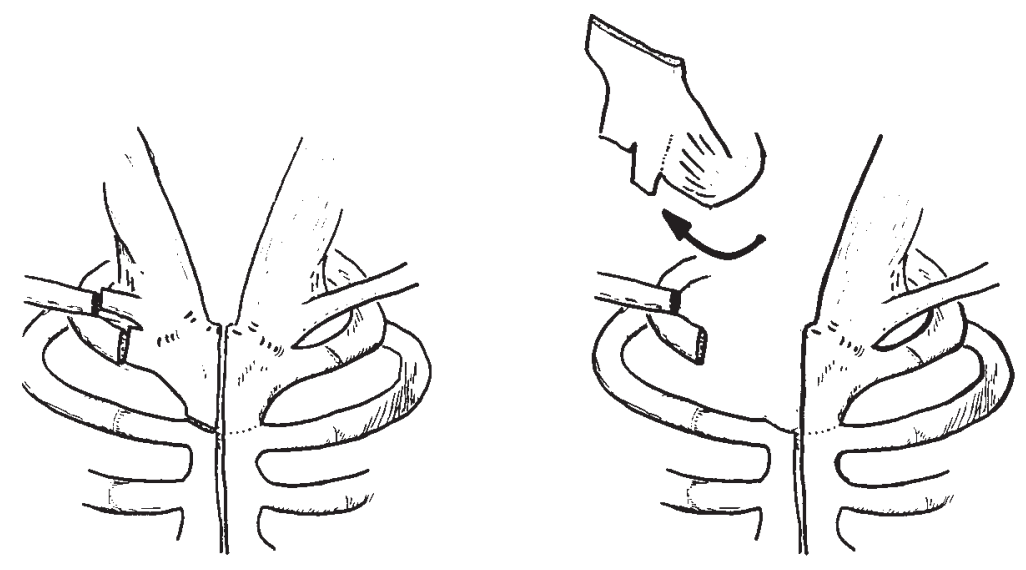

Fig 1. When a pedicled musculo-bone flap consisting of the SCM and parts of the clavicle, first rib, and sternum is turned up, broad exposure of the apex of the lung and the great vessels is gained.

Technique. The patient is placed in the supine position with the neck hyperextended and the head turned away from the operative side. A cervicothoracic incision is made from below the ear along the sternocleidomastoid muscle (SCM), the suprasternal notch, and the sternum to the xiphoid process. Median sternotomy also follows this procedure. The SCM is not divided but remains as a pedicle to be attached with the bone flap as a musculo-bone graft. This consists of the clavicle, which is divided at the third anterior portion; the sternum, which is divided just distal to the first rib attachment; and the first rib, which is divided $2 \mathrm{~cm}$ posterior to sternal attachment (Fig. 1). This modified operation was performed in 5 cases: bronchogenic carcinoma invading the superior sulcus (2 upper lobectomies and 1 sleeve upper lobectomy with pulmonary arterioplasty), invasive thymoma after radiation therapy, and liposarcoma recurrence in the right cervicothoracic region. Arterial or venous reconstruction with polytetrafluoroethylene grafts were performed in 4 cases (brachiocephalic artery, 1 case; superior vena cava, 2 cases; right innominate vein, 1 case). At reconstruction of the bone flap, sternum, and first rib were sutured with stainless steel wire. In 2 cases the clavicle was aligned with Kirschner wires; titanium-metal plates were used in the other 3 cases. Two cases of lung cancer were treated with radiation therapy (1 patient died 6 months after the operation; the other patient is alive with no cancer recurrence 18 months after the operation). A third patient died of bleeding in the pleural cavity from plural metastatic lesions 2 months after the operation.
The fourth patient (invasive thymoma) received chemotherapy and is alive 16 months after the operation. The fifth patient (recurrent liposarcoma) received radiation and chemotherapy and is alive 12 months after the operation. In all 5 cases, neither flail chest nor wound complications including muscle-bone flap necrosis occurred; but in the 2 cases in which Kirschner wires were used, fixation of the clavicle became insufficient 2 months after the operation. A fracture was observed in 1 of the cases in which a titanium-metal plate was used.

Comment. A wide operative field was gained by the use of this modified approach, especially when pulmonary hilar dissection was necessary, as in the case of upper sleeve lobectomy with pulmonary arterioplasty. Reconstruction of bone structure prevents flail chest. However, we think that a more durable titanium-metal plate should be used in clavicle fixations because we cannot expect a normal healing process in patients who are treated with radiation and/or chemotherapy soon after an operation.

\section{REFERENCES}

1. Paulson DL. Carcinoma in the superior pulmonary sulcus. J Thorac Cardiovasc Surg 1975;70:1095-104.

2. Masaoka A. Anterior approach for tumor of the superior sulcus. J Thorac Cardiovasc Surg 1979;78:413-5.

3. Dartevelle PG, Chapelier AR, Macchiarini P, Lenot B, Cerrina J, Ladurie FLR, et al. Anterior thoracic approach for radical resection of lung tumors invading the thoracic inlet. J Thorac Cardiovasc Surg 1993;105:1025-34. 